\title{
Drosophila mutational models for Huntington's disease, Parkinson's disease with dementia and levy bodies and genomic disorder Williams-Beuren syndrome
}

\author{
E.V. Savvateeva-Popova \\ Pavlov Institute of Physiology of the RAS, St. Petersburg, Russia \\ e-mail: esavvateeva@mail.ru
}

Key words: kynurenine pathway, cascade of actin remodeling, LIM-kinase 1-dependent cognitive phenotypes, hypersociability, domestication

Pavlov Institute Multi-access Center "Biocollections" exploits the concepts of N. Vavilov's (1920) Law of Homologous Rows of Hereditary Variability and T. Dobzhansky assuming that the evolutionary conservation both of gene function and of elemental behavioral mechanisms guarantees that much of what we learn in one organism will inform our understanding of behavior in all animals, including humans. This insight permits behavior-geneticists to choose organisms based on experimental tractability for a given scientific question and to develop experimental model systems to probe the causes, consequences and mechanisms of pathology leading to human disease. Traditionally, we are doing this together with Novosibirsk Institute of Cytology and Genetics, the first example is neurochemical study of the kynurenine pathway in Drosophila and the honey bee [1]. This has resulted in developing models both for Huntigton's Disease [2], aging [3] and in silico bioinformatics analysis of antioxidant properties of kynurenines, as a cause, having neurodegeneration and cataract as consequence [4]. The second example is molecular biologic study which has allowed to develop Drosophila model for genesis of LIMkinase 1-dependent cognitive phenotypes both in Parkinson's Dementia with Levy Bodies (DLB) and genomic disorder Williams-Beuren Syndrome (WBS) [5, 6]. At the same time, structural variants in genes associated with human WBS appear to underlie hypersociability in domestic dogs [7] explaining both the Pavlovian selection on different types of higher nervous activity and D. Belyaev's domestication [8].

Acknowledgements: Supported by Presidium of UB RAS (0134-2018-0003).

\section{Reference}

1. Kamyshev N.G., Savvateeva E.V., Kudriavtseva N.N., Lobacheva I.I. (1981) Serotonin content in mutants of the metabolic pathway tryptophan - xanthommatin in Drosophila melanogaster r. Dokl. Akad. Nauk USSR. 256(5):1237-1238.

2. Savvateeva E., Popov A., Kamyshev N., Bragina J., Heisenberg M., Senitz D., Kornhuber J., Riederer P. (2000) Age-dependent memory loss, synaptic pathology and altered brain plasticity in the Drosophila mutant cardinal accumulating 3-hydroxykynurenine. J Neural Transm. 107:581-601.

3. Savvateeva-Popova E.V., Popov A.V., Heinemannt, Riederer P. (2003) Drosophila Mutants of the kynurenine pathway as a model for ageing studies. Advances Experimental Medicine Biology. 527:713-22.

4. Zhuravlev A.V., Zakharov G.A., Shchegolev B.F., Savvateeva-Popova E.V. (2016) Antioxidant properties of kynurenines: density functional theory calculations. PLOS Computational Biology. DOI 10.1371/journal. pcbi.1005213.

5. Savvateeva-Popova E.V., Peresleni A.I., Scharagina L.M., Medvedeva A.V., Korochkina S.E., Grigorieva I.V., Dyuzhikova N.A., Popov A.V., Baricheva E.M., Karagodin D., Heisenberg M. (2004) Architecture of the X chromosome, expression of LIM Kinase 1, and recombination in the agnostic mutants of Drosophila: a model for human Williams syndrome. Russian Journal Genetics. 40(6):605-624.

6. Savvateeva-Popova E.V., Zhuravlev A.V., Brázda V., Zakharov G.A., Kaminskaya A.N., Medvedeva A.V., Nikitina E.A., Tokmatcheva E.V., Dolgaya J.F., Kulikova D.A., Zatsepina O.G., Funikov S.Y., Ryazansky S.S., Evgen'ev M.B. (2017) Drosophila model for the analysis of genesis of LIM-kinase 1-dependent Williams-Beuren syndrome cognitive phenotypes: INDELs, transposable elements of the Tc1/mariner superfamily and microRNAs. Frontiers in Genetics. Section Behavioral Psychiatric Genetics. 8:123. DOI 10.3389/fgene.2017.00123.

7. Von Holdt B.M., Shuldiner E., Koch I.J., Kartzinel R.Y., Hogan A., Brubaker L., Wanser S., Stahler D., Wynne C.D., Ostrander E.A., Sinsheimer J.S., Udell M.A. (2017) Structural variants in genes associated with human Williams-Beuren syndrome underlie stereotypical hypersociability in domestic dogs. Sci Adv. 19;3(7):e1700398. DOI 10.1126/sciadv.1700398.eCollection 2017.

8. Trut L., Oskina I., Kharlamova A. (2009) Animal evolution during domestication: The domesticated fox as a model. Bioessays. 31:349-360. 